\title{
Prescription, recommandation, exploration : une approche temporaliste des usages des médias socionumériques
}

Prescription, Recommendation, Exploration: A Temporalist Approach to the Uses of Social Media

Jean-Claude Domenget

\section{OpenEdition Journals}

Édition électronique

URL : http://journals.openedition.org/edc/7259

DOI : 10.4000/edc.7259

ISSN : 2101-0366

Éditeur

Université de Lille

Édition imprimée

Date de publication : 1 décembre 2017

Pagination : 125-142

ISBN : 978-2-917562-18-5

ISSN : $1270-6841$

\section{Référence électronique}

Jean-Claude Domenget, «Prescription, recommandation, exploration : une approche temporaliste des usages des médias socionumériques », Études de communication [En ligne], 49 | 2017, mis en ligne le 01 décembre 2019, consulté le 03 janvier 2020. URL : http://journals.openedition.org/edc/7259; DOI : 10.4000/edc.7259 
Prescription, recommandation, exploration : une approche temporaliste des usages des médias socionumériques

Prescription, Recommendation, Exploration: A Temporalist Approach to the Uses of Social Media 
L'état actuel des usages des médias socionumériques est marqué par une tension, entre logiques de prescription et de recommandation d'un côté, et logiques d'exploration de l'autre. Dans cet article, il s'agit d'analyser comment une approche " temporaliste », à partir de différents régimes de temporalités, permet $d$ 'interroger cette tension. Une étude de cas portant sur les formes d'interactions sociales prescrites sur les médias socionumériques, a permis de distinguer quatre régimes de temporalités (le quotidien, l'actualité, la continuité et la mise à jour). L'analyse proposée conduit à discuter la rationalité des modes d'engagement des usagers sur ces dispositifs.

Mots-clés : recommandation, prescription, exploration, média socionumérique, temporalité, usage, engagement.
Social media use is caught up in a tension between two competing logics: a logic of prescription/recommendation and a logic of exploration. In this article we explore the ways in which a "temporalist" approach allows us to better understand this tension. We identify four temporal regimes ("everyday", "current", "continuous" and "updating") which correspond to the types of social interactions prescribed on social media. Using this analytical typology of temporal regimes, we discuss different modes of user engagement on social media.

Keywords: recommendation, prescription, exploration, social media, temporalities, uses, user engagement. 
L'état actuel des usages des médias socionumériques, dont on n'évoquera jamais assez l'hétérogénéité, derrière les quelques similitudes (Stenger et Coutant, 2013), est marqué par une tension, entre logiques de prescription et de recommandation d'un côté, et logiques d'exploration de l'autre. Dans des dispositifs numériques de communication comme les médias socionumériques (de Facebook, à Twitter, de Linkedin aux forums privés ${ }^{1}$, etc.), les usages étant situés, il s'agit d'analyser notamment les caractéristiques du dispositif technique et les interactions des usagers avec les outils techniques qui le composent (Proulx, 2015). En effet, de plus en plus, des systèmes de recommandation variés (moteurs de publicités personnalisées, moteurs de recherche commerciaux, suggestions d'activités ou de contenus, etc.) accompagnent l'usager dès son inscription dans de tels dispositifs, pour lui proposer essentiellement des mises en relation, des activités à effectuer, des contenus à consulter et des offres commerciales à consommer, faisant du web social un espace communicationnel et informationnel marchand (Millerand, Proulx et Rueff, 2010). Si la pertinence de ces systèmes peut être interrogée (Domenget et Coutant, 2014), ils viennent renforcer le principal moteur de la participation dans ces dispositifs, la prescription entre pairs (Stenger, 2011).

Pourtant, face à ces usages de plus en plus prescrits, une autre logique existe, et même a toujours existé, dans I'histoire des technologies d'Internet : l'exploration. «Internet s'est fondamentalement constitué sur le réaménagement de l'action autour de l'exploration : il a donné la prééminence à l'expérimentation sur l'intériorisation, aux tâtonnements incertains sur les apprentissages formels, au jeu et au défi sur l'examen scolaire » (Auray, 2011, 330). Un tel régime exploratoire se caractérise donc par une vigilance flottante, une attention divisée et une difficulté à refréner l'excitabilité (Auray, 2016). Aujourd'hui, celui-ci est malmené. La tension se renforce avec les régimes prescriptifs et prédictifs. En effet, les usages deviennent « régis par différents algorithmes, fonctionnant comme autant d'enclosures au sein d'environnements de plus en plus fermés et propriétaires » (Ertzcheid, 2015).

L'argument principal, à l'origine de cet article, est de considérer que la tension entre ces différentes logiques peut être utilement éclairée sous l'angle des temporalités en jeu. II s'agit ainsi d'analyser comment une approche « temporaliste ", à partir de différents régimes de temporalités (Dubar, 2004), permet d'interroger cette tension entre la prescription entre pairs et les recommandations des dispositifs d'un côté, le régime exploratoire de l'autre. Plus largement,

1 Ces quatre dispositifs ont été choisis à partir de la cartographie des médias socionumériques proposée par Thomas Stenger et Alexandre Coutant (2013), entre pratiques orientées vers l'amitié et participation centrée sur l'intérêt d'un côté ; visibilité donnée à la présentation/promotion de soi et la « publication de contenus " de l'autre. Ainsi, Facebook fait partie de l'espace amitié/ soi ; les forums privés amitié/contenu ; Linkedin, intérêt/soi et Twitter, intérêt/ contenu. 
il s'agit d'interroger les modes d'engagement des usagers. Pour atteindre cet objectif, je m'appuierai sur une étude de cas ayant consisté à analyser, d'un point de vue diachronique, les formes d'interactions sociales prescrites dans les médias socionumériques (Domenget, 2014).

Dans une première partie, je présenterai ces trois régimes d'engagement en tension que sont la recommandation, la prescription et l'exploration, en soulignant les dimensions temporelles qu'ils intègrent. L'étude de cas qui a été réalisée m'a permis de distinguer quatre régimes de temporalités selon les normes d'interactions sociales repérées (le quotidien, l'actualité, la continuité et la mise à jour). Ceux-ci me conduiront à discuter la rationalité des modes d'engagement des usagers dans les médias socionumériques.

\section{1. \\ Recommandation, prescription, exploration : trois régimes d'engagement en tension}

\subsection{Les systèmes de recommandation et l'expérience des usagers}

Dans leur introduction aux systèmes de recommandation, Ghislaine Chartron et Gérald Kembellec (2014) indiquent que, selon le domaine de recherche, les points d'intérêt sur cet objet diffèrent. En informatique, la recherche reste portée sur la performance des algorithmes, leur robustesse, la conception et la comparaison de systèmes fondés sur des données sémantiques, sociales, hybrides. Les approches e-commerce s'intéressent prioritairement aux techniques permettant d'adresser de façon ciblée les produits et services aux clients potentiels. Quant au point de vue des sciences de l'information, les travaux, plus récents, pointent l'intérêt d'une prise en compte des préoccupations des usagers dans une optique d'aide à la décision. Si les recherches sur les systèmes de recommandation peuvent être teintées parfois de positivisme, dans le sens où ces outils pourraient résoudre le problème de manque d'attention et faciliter les choix (Saleh, 2014), des lectures plus critiques se font jour, interrogeant par exemple le potentiel émancipateur de tels systèmes dans la construction culturelle des individus ${ }^{2}$. Dans ce type d'approche des systèmes de recommandation, il est rappelé que la valeur qui leur est accordée par les usagers dépend de l'environnement d'accueil, de la confiance octroyée, de l'explicitation de l'opération de recommandation, etc.

En fait, cette prise en compte de l'expérience des usagers traverse aujourd'hui l'ensemble des domaines de recherche sur les systèmes de recom-

2 Voir à ce sujet la thèse en cours sur la "Sociologie des algorithmes de recommandation dans le domaine du spectacle vivant » préparée par Eloi Flesh, à I'Université d'Avignon et des Pays de Vaucluse. 
mandation. Dans une précédente étude réalisée avec Alexandre Coutant, nous étions justement partis des usages des médias socionumériques pour analyser les systèmes de recommandation de Facebook et Twitter (Domenget et Coutant, 2014). L'idée était de montrer que le changement de contexte, des sites e-commerce aux plateformes du web social, rend nécessaire quelques adaptations aux spécificités de ces dispositifs techniques. En effet, si dans le cadre d'une recherche documentaire, d'un achat, d'une pratique culturelle spécifique, etc. les systèmes mis en place reposent sur une représentation unique et précise des attentes de l'internaute, pour lesquels ils font varier les formes de prescription (Stenger, 2006), à l'inverse, dans le cadre des médias socionumériques, la question de la pertinence des systèmes de recommandation se pose pour au moins deux raisons. D'un côté, les activités auxquelles ils donnent lieu sont très diverses, parfois davantage orientées vers la sociabilité en général et non une activité précise (Ito, 2008), d'autre fois constitutives d'activités prétextes (Lahire, 2004). De l'autre, la plasticité de ces dispositifs permet la cohabitation de plusieurs figures d'usagers et rend beaucoup plus difficile la caractérisation de ceux présents (des amis avec toute l'ambiguïté plusieurs fois relevée de ce terme, des professionnels, des pairs, des collectifs en ligne, etc). Ainsi, les « amis Facebook » se révèlent être très diversifiés - des " intimes ", aux connaissances du quotidien, des membres de la famille aux célébrités, etc. (Stenger et Coutant, 2012), aboutissant à des liens aux significations tout aussi variées.

Du point de vue des temporalités en jeu, il apparaît que le régime prédictif met surtout l'accent sur les articulations des trois moments du temps que sont le présent, le passé et l'avenir. La recommandation est liée à l'anticipation des besoins, à l'aide à la décision (avenir), dans une optique d'abaissement de la surcharge cognitive et informationnelle (présent), tout en étant basée sur une analyse des comportements des individus (passé). Renforçant l'importance des usagers et complétant les formes de prescription conduites par les systèmes de recommandation, il est utile de s'intéresser également à celles en provenance des usagers eux-mêmes.

\subsection{La prescription entre pairs}

Le moteur principal de la participation, tant vantée par les discours de promotion du web 2.0, n'est autre que la prescription. Dans le cas des médias socionumériques, celle-ci prend une forme particulière due à l'importance accordée aux pairs par les usagers. Rappelons qu'Armand Hatchuel a fondé une théorie de la prescription proposant une conception originale des rapports d'influence entre acteurs d'un côté et entre acteurs et outils de gestion de l'autre (Hatchuel, 1995, 2000). Les rapports de prescription sont vus comme des sources potentielles d'influence sur les choix et activités des individus, dont les destinateurs et les formes doivent être analysés pour comprendre leur performativité. La théorie de la prescription d'Hatchuel souligne deux points fondamentaux. Une prescription demeure potentielle et suppose pour avoir 
un effet d'être reconnue. Il convient donc de comprendre les mécanismes complexes de cette reconnaissance. De plus, une prescription demeure une co-construction même si le système en est l'initiateur. L'acceptation ou le rejet de celle-ci par les usagers fournit à ce titre de précieuses informations pour optimiser ou modifier le système en fonction de sa reconnaissance.

Dans le cadre d'Internet, Thomas Stenger a adapté ce modèle au cas des interactions marchandes en ligne (Stenger, 2006). Dans ce travail, le chercheur détaille les modes de prescription proposés dans les sites marchands et souligne les rapports sur lesquels se fonde la confiance dans le système de recommandation. Pour que la prescription fonctionne, il ne suffit pas qu'elle ait été définie précisément, il faut également que la relation prescriptive soit acceptée. De notre côté, nous avons effectué une analyse énonciative des rapports de prescription en lien avec les interactions en ligne dans Facebook et Twitter, pour constater que si les systèmes de recommandation de Facebook explicitent bien la prescription qu'ils effectuent, le peu d'attention que leur accordent les usagers paraît plutôt reposer sur l'énoncé lui-même ainsi que sur la relation mise en place. Au contraire, certains systèmes de recommandation de Twitter répondent à des usages existants et leur ignorance par les usagers relèverait davantage de l'absence d'explication de ceux-ci ou d'une meilleure différenciation des logiques d'usages adressées (Domenget et Coutant, 2014). Par exemple, la logique d'usage principale des professionnels de la communication dans Twittter varie entre réseautage et curation. Cette double finalité implique que les systèmes, proposant de nouveaux abonnements, précisent la raison de leur recommandation, sous peine de se trouver en décalage avec les attentes des usagers.

La dimension temporelle de la prescription est peu présente a priori dans les analyses précédemment évoquées. La prise en compte des modalités énonciatives de la prescription, en lien avec les interactions en ligne, laisse penser que cette relation se joue principalement au niveau du quotidien, dans les pratiques d'échange via les médias socionumériques.

À l'opposé de cette complémentarité (qui reste loin d'être parfaite) entre systèmes de recommandation et logiques de prescription, un autre régime d'engagement existe : I'exploration.

\subsection{Le régime exploratoire}

Une analyse en termes d'exploration peut s'appuyer notamment sur les travaux de Nicolas Auray. Pour ce chercheur, "l'exploration n'est pas une simple passivité distraite, non focalisée, à l'événement surprenant » (Auray, 2016, 30). Elle dénote une attention flottante ou distraction émancipatrice, une flânerie productive, forme de détachement contrôlé dans le but d'une coordination attendue, d'un bienfait ultérieur, d'une cohérence de l'individu (Auray, 2016). L'engagement exploratoire se caractérise également par un état d'excitation dans lequel baigne l'individu. Ainsi, les usages des internautes peuvent relever 
d'une "logique d'exploration curieuse » et permettre des découvertes inattendues, dans un optimum magique entre la surprise recherchée et le plaisir continué (Auray, 2007).

Cependant, dans le contexte actuel du web, le régime exploratoire rentre en tension avec les régimes complémentaires de la recommandation et de la prescription. Auray a souligné depuis longtemps que l'exploration tend à être intégrée dans les systèmes de recommandation, sous la forme d'une " sérendipité encadrée " (Auray, 2007). Dans une analyse d'un site comme Delicious, il a indiqué que des techniques de sérendipité ont été déployées pour maintenir une diversité des contenus, tout en évitant le capharnaüm. Cette analyse qui montre comment le potentiel illimité de l'exploration tend à être réduit peut être mise en parallèle avec celles en termes d'émergence d'un " gouvernement des algorithmes » (Rouvroy et Berns, 2010), de développement de «bulles de filtres "(Pariser, 2012), d'« enclosures informationnelles " (Ertzcheid, 2015), lesquels enferment les individus dans leurs croyances. Ainsi, même s'« il y a toujours malgré la contre-tendance algorithmique une curiosité, une possibilité de 'sortir' volontairement de sa bulle » (Auray, 2016, 22), les logiques affinitaires contraignent beaucoup plus l'individu que les logiques de popularité (Cardon, 2015). Dans Facebook par exemple, les usagers ont doucement intériorisé le contrôle social (Kwok Choon, 2016) qui se traduit par la surveillance régulière de leur fil d'actualité ou des profils de leurs « amis ».

Les dimensions temporelles du régime exploratoire apparaissent beaucoup plus clairement. L'idée même de régime (Thévenot, 2006) l'inscrit dans la durée, dans une volonté, un projet, une envie. Elle décrit un état d'excitation, une configuration de l'action sur le modèle de l'immédiateté, même si face à une excitabilité non régulée, « il faut se donner des échéances, fixer des limites temporelles à l'activité [...]. Il faut savoir hiérarchiser ses priorités » (Auray, 2016, 37-38).

Une comparaison des interactions sociales prescrites dans les médias socionumériques va permettre de préciser les régimes de temporalités évoqués à la fin de l'analyse de chacune des notions de recommandation, prescription et exploration.

\section{2.}

\section{Une comparaison des interactions sociales prescrites dans les médias socionumériques}

Afin d'analyser la tension entre les logiques de prescription, de recommandation et d'exploration, sous l'angle des temporalités en jeu, je m'appuierai sur une étude de cas ayant consisté à analyser, d'un point de vue diachronique, 
les formes d'interactions sociales prescrites ${ }^{3}$ dans les médias socionumériques (Domenget, 2014). À partir des critères distingués par Paul M. Di Gangi et Molly Wasco (2009) et repris par Serge Proulx (2012), une analyse sociologique comparée de quatre de ces dispositifs techniques (Facebook, Twitter, Linkedin et les forums privés) a conduit à préciser les formes d'interaction produites à travers des éléments comme la nature et la qualité des dialogues, la facilité d'une accessibilité à l'autre, la possibilité d'une transparence affichée, la liberté d'une prise de risque dans l'interaction. De plus, les contenus des échanges ont été pris en compte afin d'évaluer s'ils étaient suffisamment signifiants pour les usagers. À titre d'exemple, les systèmes de recommandation sur Linkedin visent à élargir continuellement les cercles relationnels des usagers, dont les interactions sont cadrées par des algorithmes. Dans ce type de dispositif, la démarche de promotion de soi autour de centres d'intérêt s'intègre aux formes d'interaction adaptées aux normes sociales et aux cadres de référence du monde professionnel.

Pour réaliser cette comparaison, une approche « temporaliste » consiste à faire des temporalités un outil d'intelligibilité du phénomène analysé. Celles-ci renvoient à la fois aux " moments » du temps (passé, présent, avenir) et aux "domaines » de structuration du temps (travail, famille, politique, loisir, etc.) (Dubar et Rolle, 2008). Elles peuvent également être appréhendées à travers les « échelles » de temps (courte/moyenne/longue durée) et les significations associées à la gestion du temps (maîtrisé, planifié, aléas, imprévus, etc.). Une telle approche relève de plusieurs principes : il s'agit de passer d'un objet abstrait, le temps, à des objets concrets, les temporalités; de s'appuyer sur la richesse notionnelle développée par les études sur les temporalités ; d'utiliser une méthodologie qui permet d'analyser l'hétérogénéité des temporalités en jeu et de faire des temporalités un outil d'étude foncièrement interdisciplinaire. Ces différents niveaux et principes d'analyse permettent de mieux préciser les régimes de temporalités en jeu dans les régimes prédictifs, prescriptifs et exploratoires analysés.

\section{3. Quatre régimes de temporalités}

Les résultats de l'étude menée conduisent à distinguer quatre régimes de temporalités selon les normes d'interactions sociales repérées : le quotidien, l'actualité, la continuité et la mise à jour. Ces régimes de temporalités illustrent la force de prescription et de recommandation des médias socionumériques mais aussi les possibilités exploratoires existantes.

3 La notion d'interactions sociales prescrites fait ici référence à la distinction classique en sociologie des usages entre usages prescrits (ceux prévus par les concepteurs) et usages réels (ceux des usagers). 


\subsection{Le quotidien}

Le quotidien est le contexte d'étude privilégié des pratiques individuelles, sociales, professionnelles, culturelles, etc. Régime de temporalité dans lequel s'inscrivent les usages de tous les médias socionumériques, il est le cadre temporel principal pour analyser ceux de Facebook. En effet, dans ce dispositif technique, l'injonction à développer une présence en ligne repose sur le fait que " l'attractivité du site est bien cette mise en visibilité du quotidien des proches, rapporté méthodiquement par la plateforme "(Stenger, 2011, 127). Comme le précise ce chercheur, chaque usager en utilisant le service devient un " relais prescriptif ", invitant à une grande variété d'actions. II s'agit d'un " système de prescription entre amis ", d'autant plus efficace qu'il utilise un argument relationnel, «Paul - c'est-à-dire votre ami - vous suggère, vous recommande... ceci... » ou « Paul aime... cela » (Stenger, 2011, 129).

Ce système de prescription généralisée prend «forme » dans le modèle de la timeline qui peut être défini comme un flux de messages prescrivant à l'usager un engagement continu dans les relations créées via le dispositif (Domenget, 2014). Dans le cas de Facebook, l'architecture technique construite autour de ce modèle cadre un ensemble d'interactions visant à entretenir ses relations d'“ amitié " (Cadec et Proulx, 2015). De nombreuses fonctionnalités sont proposées afin d'agrandir son réseau d'amis : recherche de contacts, suggestion de comptes que l'on pourrait connaître, recommandation de comptes intéressants, accès à des listes de contacts d'autres dispositifs dont les logiciels d'emails etc. L'ensemble répond à un modèle prescrivant la mise en relation avec les " amis de nos amis ", à l'image des " contacts de nos contacts" dans Linkedin. Le contenu des échanges traduit la mise en place de routines d'interaction (prescription entre pairs, présentation de soi et gestion de sa face). Initialement rattaché à Twitter, en tant que plateforme emblématique du micro-blogging, ce modèle prescrit surtout une régularité des interactions sociales développées via ses dispositifs.

Cette régularité basée sur le quotidien des usagers est renforcée par le rôle central que jouent les systèmes de recommandation. Le modèle économique de la plate-forme repose en effet sur la valorisation d'un ciblage fondé sur l'influence du graphe social de l'individu sur ses pratiques (Mercklé, 2011). Dans cette perspective, ces systèmes ont le double rôle d'inciter les utilisateurs à participer, en leur recommandant des activités susceptibles de les intéresser, et d'exploiter les relations nouées pour effectuer des prescriptions commerciales adaptées. Cependant, de fortes divergences existent entre les logiques de ces systèmes et celles des activités. Elles concernent aussi bien la nature des liens, au sein des types de collectifs formés par chaque réseau social : famille, collègues, amis, partenaires sportifs, etc., que le contexte dans lequel se déroulent les activités (humour, ironie, " private joke », etc.) (Domenget et Coutant, 2014). 
Ainsi, dépassant les visions routinières de la vie quotidienne, cette analyse du quotidien peut être rapprochée de celle de Claude Javeau autour d'un « bricolage du social » (2001) ou avant lui de Michel de Certeau, à travers ses " arts de faire » (1990). Au-delà des bulles algorithmiques, le quotidien est un terreau aux tâtonnements, essais, erreurs et tests, traduisant l'importance du régime exploratoire dans ces activités liées à la sociabilité.

\subsection{L'actualité}

Le suivi de l'actualité concernant ses centres d'intérêt est l'un des usages dominants des médias socionumériques. Il concerne aussi bien des informations d'ordre journalistique (de nature politique, sociale, culturelle, etc.) que celles d'ordre professionnel, participant d'un travail de veille (nouveautés, analyses, bonnes pratiques, etc.). Depuis les réflexions de Jean-François Tétu (1993) sur l'actualité, "comme impasse du temps ", renvoyant à une idée de coprésence et de direct, ces " marqueurs » de " moments » du temps (présent, passé, futur) font écho à la notion de temps réel. Or, le temps réel réduit l'actualité à sa valeur d'usage éphémère, dont l'importance est mesurée par le temps (durée) qui lui est accordé.

Sans être bien sûr le seul dispositif du web social à se baser sur le temps réel, Twitter est symptomatique de l'intégration de ce régime de temporalité dans les interactions prescrites, à travers sa double dimension de réseau social et de média de diffusion d'informations (Kwak et al., 2010). Cette dimension du temps réel et le faible espace de présentation de soi que constitue la biographie dans un dispositif comme Twitter donnent lieu à une réduction du contexte (context collapse) (Marwick et boyd, 2010) pour interpréter les messages. Ainsi, les systèmes de recommandation qui se sont progressivement greffés aux usages de Twitter, dans le but de faciliter l'accessibilité à l'autre, élargir les cercles d'abonnements et multiplier les interactions, ne s'avèrent pas toujours adaptés. En effet, la prescription entre pairs est au cœur des usages de ce dispositif. Elle s'appuie sur une syntaxe propre, une énonciation tournée vers la thématisation des comptes (leur positionnement) et des pratiques de suggestions de comptes (notamment \#FF) remédiant à l'absence initiale d'un système de recommandation officiel (Domenget, 2014). Depuis lors, les systèmes de recommandation invitant à suivre tel ou tel compte ne tirent pas parti de la dynamique de prescription entre pairs, en associant à l'énoncé de la prescription sa position énonciative de plateforme. Les pairs, substrats énonciatifs permettant la reconnaissance, ne sont effectivement pas convoqués explicitement dans les propositions qu'elle formule.

Dans le suivi d'une actualité, centrée sur ses centres d'intérêt, le travail de filtrage de l'information voire de curation sociale proposé par de nombreux usagers se double d'un objectif de réseautage. Cette double finalité implique que le système de recommandation, proposant de nouveaux abonnements ou mettant en avant certains messages, explicite la logique de la prescription 
effectuée. En effet, pour éviter un enfermement dans une «bulle informationnelle » et la reprise d'une même information, allant à l'encontre d'une logique de curation, l'algorithme de recommandation doit être source d'une ouverture thématique et relationnelle, proposer une diversité de contenus, objet d'une "logique d'exploration curieuse ».

\subsection{La continuité}

La continuité est un régime de temporalité qui peut être plus difficile à saisir dans sa logique de prescription des interactions sociales. Elle s'oppose à la nouveauté ou à la rupture afin de souligner une des richesses des médias socionumériques, celle de permettre le maintien d'une relation même en cas d'absence d'échanges réguliers. Très liée à des logiques d'usage de sociabilité, la continuité se traduit par des activités (publication liée à son quotidien, demande de nouvelles, etc.) afin de " rester, garder, reprendre contact », de " maintenir le lien », etc.

La continuité est le régime dominant des sites de réseautage professionnel. Entre volonté de maintenir des liens forts et exploiter «la coopération des liens faibles » (Aguiton et Cardon, 2007), I'activité sur Linkedin ou Viadeo passe par une logique de continuité dans le développement et l'entretien de son réseau de contacts. Dans cette "famille " des sites de réseautage ou réseaux socionumériques professionnels, la démarche de promotion de soi, autour de centres d'intérêts, s'intègre dans des formes d'interaction adaptées aux normes sociales et aux cadres de référence du monde professionnel. Si l'accessibilité à l'autre est limitée pour favoriser les abonnements aux offres payantes, les interactions dans ces dispositifs sont marquées par l'objectif de créer des opportunités professionnelles, d'où une nature et une forme de dialogue très sérieuses. La présence d'un quasi-CV conduit à afficher une transparence importante vis-à-vis de son identité réelle, limitant d'autant plus la prise de risque dans l'interaction.

Sans que les usagers en aient forcément conscience, les possibilités d'interactions offertes par ces dispositifs dépendent d'un filtrage des comptes puis des messages, issu d'algorithmes avancés (Cardon, 2015). Une participation régulière est ainsi prescrite, consistant à partager des contenus ou à modifier les informations de son profil afin d'apparaître dans le fil d'actualité de ses contacts. Dans le cas de Linkedin, cette forme de prescription est complété par un système de recommandation d'action qui s'appuie sur des formes d'énonciation incitant à élargir son cercle relationnel et à renforcer ses centres d'intérêts : "les connaissez-vous? ", " qui a consulté votre profil ?", " qui a consulté vos nouvelles? ", "les groupes que vous pourriez aimer ", "les entreprises que vous pourriez vouloir suivre ". Notons également que pour ressortir dans les classements, outre le soin particulier à apporter à son profil, une participation régulière aux groupes de discussion importe, laquelle permet de réduire la distance de relation avec les membres de ce groupe. 
Là encore, face à cette tendance dominante d'une généralisation d'une prescription d'activités régulières, renforcée par un système de recommandation ayant intégré la distinction entre la force des liens entre les usagers, « la force des coopérations faibles » est un moteur d'activités pour l'exploration.

\subsection{La mise à jour}

La mise à jour et l'attente des modifications apportées par les autres usagers caractérisent les interactions sur les dispositifs socionumériques. Elles peuvent se traduire par le suivi de sollicitations ou d'alertes avec des outils comme Tweetdeck ou Hootsuite. Le caractère incertain des mises à jour des contenus renvoie à la dimension asynchrone ou synchrone de ces dispositifs. La synchronie caractérise les modalités d'interactions développées dans de nombreux dispositifs, dont les messageries instantanées. Elle a été beaucoup étudiée lors de l'émergence des recherches sur la communication médiatisée par ordinateur (CMO) à la fin des années 1990, et s'est généralisée avec l'essor des médias socionumériques. De nombreuses recherches, intégrant la dimension synchronique des interactions, s'inscrivent dans une approche ethnométhodologique s'intéressant par exemple aux formes de présence à distance (Licoppe, 2012). Outre le démarrage souvent non prévu de l'échange, la synchronie pose la question du rythme de cet échange.

En tant que dispositifs asynchrones, les forums (privés comme publics) apparaissent comme appartenant à une autre génération, n'étant pas basé sur le régime du temps réel. Un système de classement des contenus très avancé aide l'usager à évaluer la pertinence de la mise à jour d'une discussion au sein des différentes catégories de messages. Le design de l'interface et les fonctionnalités, sur le modèle du phpBB (bulletin board open source), permettent de mettre en avant certains messages (annonces et post-it), en les maintenant en haut du classement quelle que soit leur antériorité, bien avant les modèles de la timeline de Facebook ou Twitter. D'autres fonctionnalités sont présentes afin d'informer l'usager des sujets en cours, de ceux qu'il n'a pas encore consultés, des commentaires apportés par les autres membres à ses messages, etc. Les forums s'organisent autour d'un système hiérarchique, distinguant différents statuts, plus poussé que dans d'autres dispositifs plus récents. La prescription touche ainsi aussi bien les types de contenus que les comportements. La liberté dans l'interaction est encadrée par une modération et une surveillance par les membres (normes d'interaction fortes, rappel à l'ordre, déplacement de sujets dans d'autres catégories, suppression de messages, fermeture d'un compte, etc.). Car parmi les membres d'un forum, les contributeurs côtoient également des leechers qui ne font que télécharger et des trolls qui viennent "polluer " les fils de discussion. Basées beaucoup plus sur le modèle de la conversation (Marcoccia, 2004) que d'autres dispositifs, les interactions sur les forums laissent place à des formes d'exploration, dépendant des autres usagers. Le caractère incertain, aléatoire de la mise à jour des fils de discussion conduit à d'autres formes d'engagement, entre flânerie curieuse et engagement 
soutenu, donnant lieu à une satisfaction ou insatisfaction liées à l'attente et à l'importance de cet engagement.

En résumé, ces différents régimes de temporalités (quotidien, actualité, continuité, mise à jour) illustrent les tensions existantes entre les régimes prescriptifs, prédictifs et exploratoires dans les interactions en ligne. Ils soulignent la force de prescription entre usagers et de recommandation des systèmes mis en place dans les médias socionumériques, tout pointant les marges de manœuvre exploratoires laissées aux usagers. Cette analyse en termes de contraintes et de marges de liberté peut être complétée par un questionnement autour de la rationalité derrière ces régimes d'engagement.

\section{4. \\ Quelle rationalité derrière ces régimes d'engagement?}

Plus qu'une interrogation sur les conditions de l'efficacité et de l'effectivité relatives des prescriptions et recommandations, c'est la question des modes d'engagement des usagers qui sera discutée dans cette conclusion. Car si des normes sociales et d'interactions dans les médias socionumériques sont aujourd'hui bien ancrées dans les usages (de la prescription ordinaire et la gestion des relations d'amitié sur Facebook, au maintien d'un lien fort au sein des forums privés), des tactiques de résistance (relevant du régime exploratoire) persistent encore et ne doivent pas être négligées. Ce sont elles qui sont le plus souvent à la source des changements de pratiques.

Les tensions entre les régimes prescriptifs, prédictifs et exploratoires analysées sous l'angle des temporalités peuvent être également questionnées à travers les régimes d'attention induits (Boullier, 2014). Le modèle généralisé de la timeline conduit à proposer deux régimes attentionnels aux usagers. L'alerte relève d'un régime centré sur l'intensité. Focalisée sur l'instant, l'attention-alerte est fondamentalement perturbatrice, elle déconcentre. Elle est source d'une fragilité de l'attention, potentiellement riche de découvertes, dans une logique d'exploration. Régime d'attention dominant de Twitter, elle s'oppose à l'immersion, qui illustre une forte irréversibilité (autre dimension temporelle) car elle demande du temps pour être expérimentée pleinement. Immersive, l'expérience de Facebook peut tendre vers l'addiction. La fidélisation correspond à un autre régime attentionnel, marqué par la durée. Sur Linkedin, la fidélisation vise une sorte d'immunité pour protéger des agressions tentées par les capteurs d'attention concurrents. Quant à la projection, elle traduit une volonté de projeter ses propres cadres et modèles sans se laisser affecter par d'autres éléments. Elle correspond au régime attentionnel dominant sur les forums privés, rendu possible par l'asynchronie de ces dispositifs. Ainsi, l'analyse des régimes d'attention développés sur les médias socionumériques 
permet d'illustrer les tensions entre les trois régimes d'engagement distingués. D'un côté l'immersion et la fidélisation renvoient à des valeurs d'irréversibilité et de durée (donc de stabilisation des pratiques correspondant aux régimes prescriptifs et prédictifs) et de l'autre, l'alerte relève d'une intensité perturbatrice (donc d'une fragilité des usages correspondant au régime exploratoire). Quant à la projection, elle laisse place aux choix des usagers et ouvre la question de la rationalité des modes d'engagement dans les activités en ligne, laquelle est loin d'être uniforme sur un modèle de volonté, de responsabilité et d'autonomie.

Pour conclure, le régime exploratoire, tel qu'il a été analysé par Nicolas Auray, renvoie à un modèle d'engagement autour d'un projet, d'une envie, d'une volonté. Il peut être utilement complété par le concept de sérendipité en lien avec les temporalités ${ }^{4}$. A partir d'une première acception comme « la faculté de découvrir par hasard et sagacité des choses que l'on ne cherchait pas " (Catellin, 2014 , 25), le concept de sérendipité a parcouru tous les domaines du savoir et il est devenu synonyme de découverte accidentelle, trouvaille heureuse, coïncidence, etc. Le développement d'Internet, conduisant les chercheurs à travailler sur la possible programmation de la sérendipité, renvoie à la recherche de la diversité dans l'approche des systèmes de recommandation en informatique. Mais c'est bien sûr en lien avec le concept d'exploration que la sérendipité a le plus d'intérêt. Il s'agit alors de poursuivre la voie ouverte par Nicolas Auray, analysant certains comportements essentiels de la vie (apprendre, travailler, faire des rencontres et se détendre) sous l'angle de l'ouverture attentionnelle, de l'exploration, de la curiosité et de la sérendipité. 
Bibliographie

Aguiton C. and Cardon D. (2007). " The strength of weak cooperation: an attempt to understand the meaning of Web2.0 ". In Communications and Strategies, $n^{\circ} 65$, p. 51-65.

Auray N. (2007). " Folksonomy: The New Way to Serendipity ". In Communications and Strategies, $n^{\circ} 65$, p. 67-91.

Auray N. (2011). " Les technologies de l'information et le régime exploratoire ". In Van Andel P. et Bourcier D. (dir.), La sérendipité : le hasard heureux, Paris, Hermann, p. 329-343. Disponible sur http:// ses-perso.telecom-paristech.fr/ auray/2010AuraySerendipite.pdf (page consultée le 15 mars 2017).

Auray N. (2016). L'Alerte ou l'Enquête. Une sociologie pragmatique $d u$ numérique, Paris, Presses des Mines.

Boullier D. (2014). « Médiologie des régimes d'attention ». In Citton Y. (dir.), L'économie de l'attention. Nouvel horizon du capitalisme?, Paris, La Découverte, p. 84-108.

Cadec K. et Proulx S. (2015). " Les représentations de l'amitié sur Facebook. Un continuum hors ligne/en ligne ". In Communication, vol. 33/2. Disponible sur http:// communication.revues.org/5755. (page consultée le 15 mars 2017). doi : 10.4000/communication.5755
Cardon D. (2015). À quoi rêvent les algorithmes. Nos vies à l'heure des big data, Paris, Seuil.

Catellin S. (2014). Sérendipité. Du concept au conte, Paris, Éditions du Seuil.

Certeau de M. (1990). L'invention du quotidien, tome 1 : arts de faire, Paris, Gallimard.

Chartron G. et Kembellec G. (2014). "Introduction générale aux systèmes de recommandation ». In Kembellec G., Chartron G. et Saleh I. (dir.), Les moteurs et systèmes de recommandation, Londres, ISTE éditions, p. 23-42.

Di Gangi P. M. and Wasco M. (2009). "The Co-creation of Value: Exploring User Engagement in User-Generated Content Websites ». In Sprouts: Working Papers on Information Systems, vol. 9, $\mathrm{n}^{\circ} 50$.

Domenget J.-C. (2014). " Une comparaison des interactions sociales prescrites sur les médias sociaux $"$. In

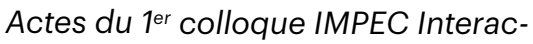
tions Mutlimodales Par Ecran, Lyon, p. 293-307. Disponible sur https:// impec.sciencesconf.org/conference/ impec/pages/Impec2014_Domenget. pdf (page consultée le 15 mars 2017).

Domenget J.-C. et Coutant A. (2014). " Partir des usages pour analyser les systèmes de recommandation : le cas des médias sociaux ». In Kembellec G., Chartron G. et Saleh I. (dir.), Les moteurs et systèmes de recommandation, Londres, ISTE éditions, p. 43-67. 
Dubar C. (2004). "Régimes de temporalités et mutation des temps sociaux ». In Temporalités, $\mathrm{n}^{\circ} 1$. Disponible sur http://temporalites.revues.org/661 (page consultée le 15 mars 2017). doi : 10.4000/temporalites.661

Dubar C. et Rolle C. (2008). « Les temporalités dans les sciences sociales : introduction ». In Temporalités, $n^{\circ} 8$. Disponible sur http://temporalites.revues.org/57. doi : 10.4000/temporalites.57

Ertzcheid O. (2015). «Usages de l'information numérique : comprendre les nouvelles enclosures algorithmiques pour mieux s'en libérer ". In Revue française des sciences de l'information et de la communication, $\mathrm{n}^{\circ} 6$. Disponible sur http://rfsic.revues.org/1425 (page consultée le 15 mars 2017). doi :10.4000/rfsic.1425

Hatchuel A. (1995). « Les marchés à prescripteurs ». In Verin $\mathrm{H}$. et Jacob A. (dir.). L'inscription sociale du marché, Paris, L'Harmattan, p. 205-225.

Hatchuel A. (2000). "Quel horizon pour les sciences de gestion? Vers une théorie de l'action collective ». In David A., Hatchuel A. et Laufer R. (dir.). Les nouvelles fondations des sciences de gestion, Paris, Vuibert, p. 7-43.

Ito M. et al. (2008). Living and learning with new media: summary of findings from the digital youth project, Berkeley, Rapport. Disponible sur http://digitalyouth. ischool.berkeley.edu/files/report/
digitalyouth-WhitePaper.pdf (page consultée le 15 mars 2017).

Javeau C. (2001). Le bricolage du social. Un traité de sociologie, Paris, PUF.

Kwak H., Lee C, Park H. and Moon S. (2010). "What is Twitter, a Social Network or a News Media ? ». In Proceedings of WWW'10, NewYork, p. 591-600. Disponible sur https://an.kaist.ac.kr/ haewoon/ papers/2010-www-twitter.pdf (page consultee le 15 mars 2017).

Kwok Choon M. J. (2016). « La déconnexion temporaire à Facebook : entre le FOMO et intériorisation douce du contrôle social ». In Terminal, $n^{\circ} 118$. Disponible sur https://terminal.revues.org/1447 (page consultée le 20 juin 2017).

Lahire B. (2004). La culture de I'individu, Paris, Nathan.

Licoppe C. (2012). « Les formes de la présence ". In Revue française des sciences de l'information et de la communication, $\mathrm{n}^{\circ} 1$. Disponible sur http://rfsic.revues. org/142. (page consultée le 15 mars 2017). doi : 10.4000/rfsic.142

Marcoccia M. (2004). "L'analyse conversationnelle des forums de discussion : questionnements méthodologiques ". In Les Carnets du Cediscor, $\mathrm{n}^{\circ}$ 8. Disponible sur http://cediscor.revues.org/220 (page consultée le 15 mars 2017).

Marwick A. and boyd E. (2010). «I tweet Honestly, I Tweet Passionetely: 
Twitter Users, Context Collapse and the Imaginated Audience ". In New Media Society, vol. 13, n 1, p. 114-133.

Mercklé P. (2011). Sociologie des réseaux sociaux, Paris, La Découverte.

Millerand F., Proulx S. et Rueff J. (2010). Web social, mutation de la communication, Québec, PUQ.

Pariser E. (2012). The Filter Bubble: How the New Personalized Web Is Changing What We Read and How We Think, New York, Penguin Books.

Proulx S. (2012). « L'irruption des médias sociaux : enjeux éthiques et politiques ». In Proulx S., Millette M. et Heaton L. (dir.). Médias sociaux : enjeux pour la communication, Québec, PUQ, p. 9-31.

Proulx S. (2015). " La sociologie des usages, et après ? ». In Revue Française des Sciences de I'Information et de la Communication, $\mathrm{n}^{\circ}$ 6. Disponible sur https:// rfsic.revues.org/1230 (page consultée le 20 juin 2017).

Rouvroy A. et Berns T. (2010). " Le nouveau pouvoir statistique. Ou quand le contrôle s'exerce sur un réel normé, docile et sans événement car constitué de corps 'numériques'... ». In Multitudes, $n^{\circ} 40$, p. 88-103. Disponible sur http://www.cairn.info/ revue-multitudes-2010-1-page-88. htm doi : 10.3917/mult.040.0088

Saleh I. (2014). « Avant-propos. Les moteurs et systèmes de recommandation ». In Kembellec G., Chartron G. et Saleh I. (dir.), Les moteurs et systèmes de recommandation, Londres, ISTE éditions, p. 17-21.

Stenger T. (2006). « La prescription dans le commerce en ligne : proposition d'un cadre conceptuel issu de la vente de vin par Internet ». In Revue Française du Marketing, $\mathrm{n}^{\circ}$ 209, p. 71-85.

Stenger T. (2011). « La prescription ordinaire : dynamique de l'action collective sur les réseaux socionumériques ». In Hermès, n 59, p. 125-132.

Stenger T. et Coutant A. (2012). « Un monde d'amis ? Une ébauche de typologie sur les réseaux socionumériques ». in Proulx $S$. et Klein A., Connexions : communication numérique et lien social, Namur, PUN, p. 213-228.

Stenger T. et Coutant A. (2013). «Médias sociaux : clarification et cartographie. Pour une approche sociotechnique ». In Décisions marketing, $\mathrm{n}^{\circ} 70$, p. 107-117.

Tétu J.-F. (1993). « L'actualité, ou l'impasse du temps ». In Bougnoux D. (dir.), Sciences de l'information et de la communication. Paris, Larousse, p. 713-722.

Thévenot L. (2006). L'action au pluriel. Sociologie des régimes d'engagement, Paris, La Découverte. 
